\title{
THE GEO-DSCM SYSTEM AND ITS APPLICATION TO THE DEFORMATION MEASUREMENT OF ROCK MATERIALS
}

\author{
Shao-Peng Ma ${ }^{1}$, Xiang-Hong $\mathrm{Xu}^{2}$, Yong-Hong Zhao ${ }^{3}$ \\ 1) Dept. of Geophysics, Peking University, Beijing, 100871, China \\ masp@pku.edu.cn \\ $\left.{ }^{2}\right)$ LNM, Institute of Mechanics, Chinese academy of Sciences, Beijing, 100083, China \\ xxh@Inm.imech.ac.cn \\ $\left.{ }^{3}\right)$ Dept. of Geophysics, Peking University, Beijing, 100871, China \\ zhyh@pku.edu.cn
}

\begin{abstract}
Digital Speckle Correlation Method (DSCM) is a useful tool for whole field deformation measurement, and has been applied to analyze the deformation field of rock materials in recent years. In this paper, a Geo-DSCM system is designed and used to analyse the more complicated problems of rock mechanics, such as damage evolution and failure procedure. A weighted correlation equation is proposed to improve the accuracy of displacement measurement on a heterogeneous deformation field. In addition, a data acquisition system is described that can synchronize with the test machine and can capture speckle image at various speeds during experiment. For verification of the Geo-DSCM system, the failure procedure of a borehole rock structure is inspected and the evolution of the deformation localization is analysed. It is shown that the deformation localization generally initializes at the vulnerable area of the rock structure but may develop in a very complicated way.
\end{abstract}

Key words: Digital speckle correlation method (DSCM), deformation measurement, whole-field, rock failure

\section{INTRODUCTION}

Deformation measurement is of great importance to experimental study in rock mechanics, and also to further numerical and theoretical analysis. With the result of deformation measurement, the basic mechanical constant, the procedure of damage evolution and failure could be analysed. The most commonly used technique for deformation measurement in rock mechanics is the strain gauge method. Because of the heterogeneity of the mechanical properties of rock materials, this point-wise measurement technique sometimes failed in revealing the global response of the specimen to loads, especially during loading. In recent years, optical methods such as holography, morie metrology and speckle interferometry are applied to obtain the whole field deformation of rock materials, e.g. Huang et al (1990) and Berthaud et al (1997). Among these, Digital Speckle Correlation Method (DSCM), e.g. Peters and Ranson (1981), is a frequently used method.
Several advantages of this method are important to the experimental study of rock mechanics. Firstly, the experiment using DSCM could be completed in a complex environment where the other interferometry optical methods are impossible to use. Secondly, DSCM is convenient to use for experiments with specimens of different sizes, because its sensitivity is adjustable by changing the magnification of imaging system. Finally, the experiment is easy to do and is very cheap. This allows many repetitions of tests to be done, which is very helpful for rock mechanics study.

DSCM is already used in deformation measurement of rock and rock-like materials by some researchers in recent years, e.g. Zhao et al. (2002). Although these studies showed the advantages, DSCM encounters some new problems that are always ignored in experiments focusing on other materials. Firstly, the accuracy of displacement measurement will decrease greatly at the location where deformation field is greatly 
heterogeneous, because the currently used correlation equations treat all the pixels within the correlation window equally and this must decrease the spatial resolution of the displacement measurement result. Secondly, current DSCM systems are not designed according to the characteristics of the experiment of rock mechanics. It is not suitable to inspect the whole deformation and failure procedure of rock materials.

In this paper, a Geo-DSCM system is developed and realized by improving DSCM on the consideration of the characteristics of the deformation measurement of rock materials. A weighted correlation equation and a speckle image acquisition system is designed and realized. With this Geo-DSCM system, the damage evolution and failure procedure of rock materials could be traced and analyzed using the deformation field obtained.

\section{PRINCIPLE OF DSCM AND THE WEIGHTED CORRELATION EQUATION}

\subsection{Principle of DSCM}

The principle of DSCM could be simply shown as Fig.1 (take the displacement measurement of a point $P$ as an example): the speckle pattern $f$ is firstly selected from the source image, then the pattern $f$ is to be matched on the target speckle image. The index point $P$ ' of the matched pattern $g$ is thought as the position of $P$ after deformation and the coordinates differences $(u, v)$ of $P$ and $P$, indicate the displacement. The correlation coefficient of these two patterns is defined as the criterion to determine the optimum match of the speckle patterns. Eq.1 is a typically used correlation equations.

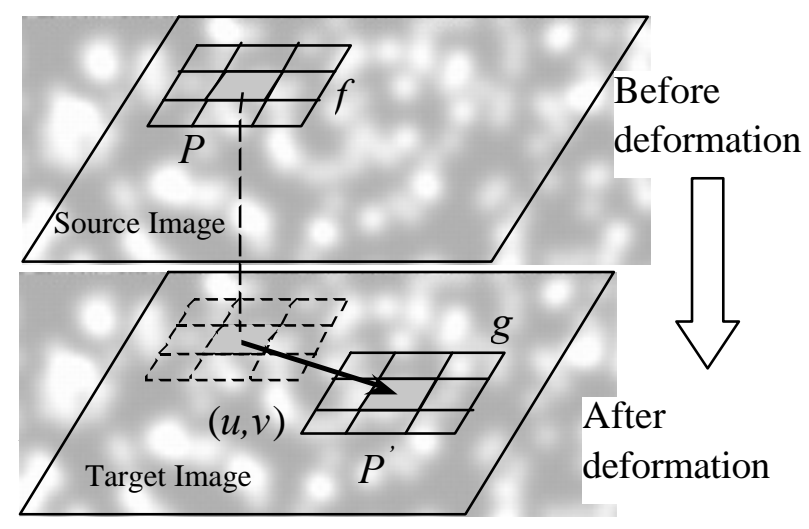

$$
C=\frac{\sum \sum\lfloor(f-\bar{f}) \cdot(g-\bar{g})]}{\left[\sum \sum(f-\bar{f})^{2} \cdot \sum \sum(g-\bar{g})^{2}\right]^{1 / 2}}
$$

The window to crop the source pattern $f$ and target pattern $g$ is often named as correlation window. This window is often square, generally with the size of $20 \times 20 \sim 50 \times 50$ pixel square. For precision measurement, the pixel level search as shown in Fig.1 is not enough and the sub-pixel level search should be completed by interpolation, iterative or fitting, e.g. Sutton et al. (1988) and Tian et al. (1986). With the better control of the quality of the speckle image and the appropriate selection of the processing algorithm, the accuracy for displacement measurement is expected to be 0.01 pixels, e.g. Zhou and Goodson (2001).

All the processing algorithms of DSCM, the correlation-search, the sub-pixel estimation, are based on the calculation of the correlation coefficient $C$. However, as shown in Fig.1 and Eq.1, the calculation of $C$ is an area-based operation. The $C$ value of one point is not determined separately by the gray value of that point, but by the gray values within an area, the correlation window. This operation is equivalent to performing a smoothing operation to the randomly distributed speckle image. The much smoother distribution of the correlation coefficients comparing to the distribution of the gray value of speckle image verifies this smoothing operation. That is, the spatial resolution of DSCM depends on the spatial frequency of the distribution of $C$ (finally depends on the size of correlation window), which is much lower than the size of pixel. Therefore, it can be concluded that the resolution of DSCM is far from the pixel size. Considering of the low spatial resolution, the accuracy of DSCM must decrease greatly if a much heterogeneous deformation field is to be measured.

\subsection{The weighted correlation equation}

According to the principle of DSCM, the speckle pattern represents the information of one point (generally the center of the correlation window), it is reasonable that different pixel in speckle pattern takes different weight on calculating of correlation coefficient $C$. If an appropriate weighting method is applied, the resolution of DSCM is expected to be enhanced and the accuracy of deformation measurement is improved when non-homogenous deformations exist. 
One of the simple weighting methods is distance-related approach. A weighted correlation equation using distance-related weighting method is defined as Eq.2.

$$
C_{w}=\frac{\left\{\sum \sum[w(\xi, \eta)(f-\bar{f}) \cdot w(\xi, \eta)(g-\bar{g})]\right\}^{2}}{\sum \sum w^{2}(\xi, \eta)(f-\bar{f})^{2} \cdot \sum \sum w^{2}(\xi, \eta)(g-\bar{g})^{2}}
$$

where

$$
\left\{\begin{array}{c}
w(\xi, \eta)=\frac{1}{\left(\xi^{2}+\eta^{2}\right)^{p}} \\
w(0,0)=1
\end{array}\right.
$$

is a distance-related weighting function, $\xi, \eta$, the orthogonal coordinates with origins at the center of the speckle pattern $f$, are shown in Fig. 2 .
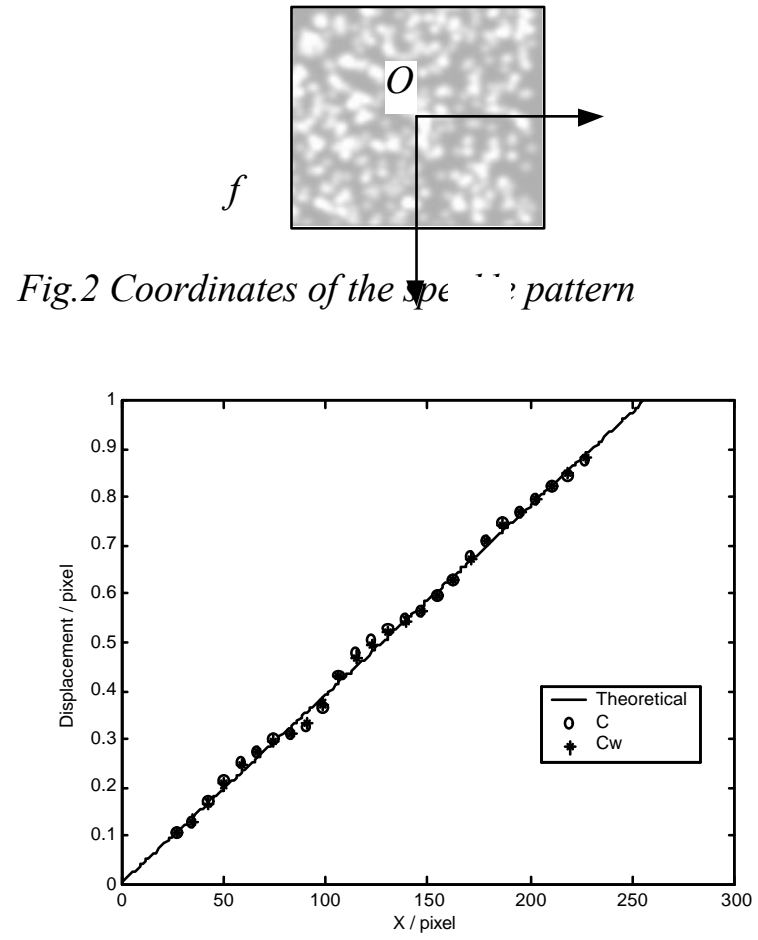

(a) result of the homogenous deformation

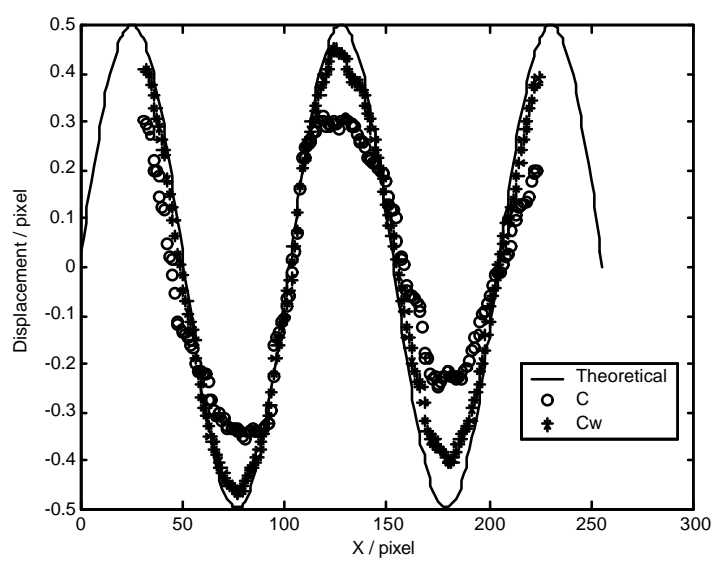

(b) result of the heterogeneous deformation

Fig.3 Effect of the weighted correlation equation $(\mathrm{p}=0.25)$

To verify the improvement of the accuracy on displacement measurement of the weighted correlation equation in Eq.2, a simulated experiment (1-D experiment for simplicity) was completed. A 1-D speckle signal (taken from a section of a real speckle image) is firstly applied with homogenous deformation and heterogeneous deformation respectively with numerical method and then processed with 1-D DSCM completely samilar to the 2-D DSCM algorithm expect that 1$\mathrm{D}$ signal is processed), using the ordinary correlation equation and the new weighted correlation equation. The result displacement is compared with the theoretical displacement (Fig.3). As shown in the Figures, for the homogenous deformation, the weighted correlation equation has almost no effect on accuracy improvement, but it greatly improves the accuracy on heterogeneous deformation measurement. Actually, the spatial frequency of the homogenous displacement field is quite low, but the spatial frequency of the heterogeneous displacement field is relative high. The difference between the measured displacement fields is the reason of the difference of the effectiveness of the weighted correlation equation.

\section{DATA ACQUISITION SYSTEM}

Speckle image is the foundation of DSCM processing, so the acquisition of enough images during the loading of the rock specimen is of great importance to analysis of the whole deformation procedure of rock materials. Considering the characteristics of the deformation and failure of rock materials, the experimental environment and the image acquisition, storage and processing ability of currently used equipment, a data acquisition system as Fig.4 is designed for GeoDSCM.

During experiment, the load signal is led out from the test machine and obtained by the imagecapturing computer after converting by an A/D card. The computer then determines the deformation stage of the loaded specimen according to the load signal and adjusts the image capturing speed according to the previous arrangement. At the early loading stage, the deformation is much slower and homogenous, and the speckle images are captured with a quite lower 
speed. While, at the later deformation stage, especially on the event of the catastrophic failure, the deformation is much faster and heterogeneous, the speckle images must be captured with much higher speed, otherwise the information before failure will be lost. The load signal, the captured speckle images and the time where the image is captured are all stored in the computer. When the loading is completed, the images are analyzed using DSCM software and its result could be analyzed corresponding to the result of other inspection, for example the $\mathrm{AE}$, after synchronizing with those inspection systems by the time-load curve. The control of the image capturing speed ensures that the system can capture enough "useful" images with the current image capturing equipment.

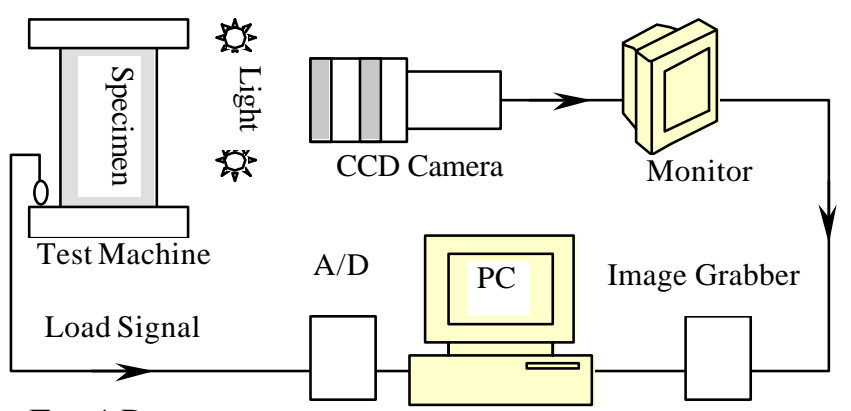

Fig.4 Data acquisition system

\section{EXPERIMENT}

To verify the effect of the Geo-DSCM system, the deformation procedure of a borehole rock structure is measured using this system. Dimensions of specimen and experiment arrangement are shown in Fig.5. The specimen is made of marble, with the elastic modulus of $30 \mathrm{Gpa}$, Possion's ratio of 0.25 . Before experiment, the surface of the specimen is processed to bear the random speckle pattern. The specimen is compressed on a MTS 810 test machine with the load speed of $0.02 \mathrm{~mm} / \mathrm{min}$. The loading curve (load-displacement curve) is shown as Fig.6. The speckle images are captured with the time interval of $10 \mathrm{~s}$ when load is less than $10 \mathrm{KN}$, interval of $1 \mathrm{~s}$ when load is between $10 \mathrm{KN}$ and $20 \mathrm{KN}, 500 \mathrm{~ms}$ when load between $20 \mathrm{KN}$ and $25 \mathrm{KN}$, and $80 \mathrm{~ms}$ when load is larger than $25 \mathrm{KN}$. Totally 4 experiments are completed, about 800 speckle images are captured per specimen. The length-pixel ratio of the imaging system is $0.0568 \mathrm{~mm} / \mathrm{pixel}$.

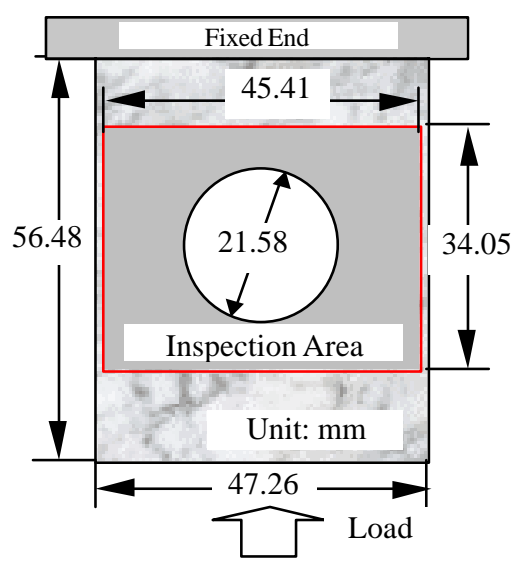

Fig.5 Specimen and arrangement of Experiment

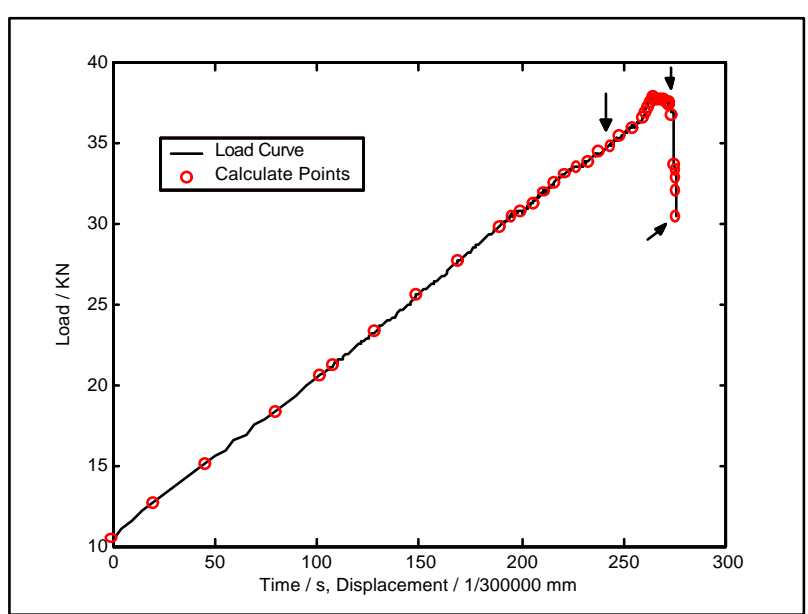

Fig.6 Load curve of the specimen

After experiment, the speckle images are analyzed with DSCM and the displacement field and strain field during all the loading procedure are obtained. Fig. 7 shows 3 stages (indicated in Fig.6) of the evolution of the deformation of No.2 specimen (results of the other 3 specimen are similar). Thanks to the whole field and throughout loading deformation result from Geo-DSCM system, the complicated evolution procedure could be clearly revealed. 


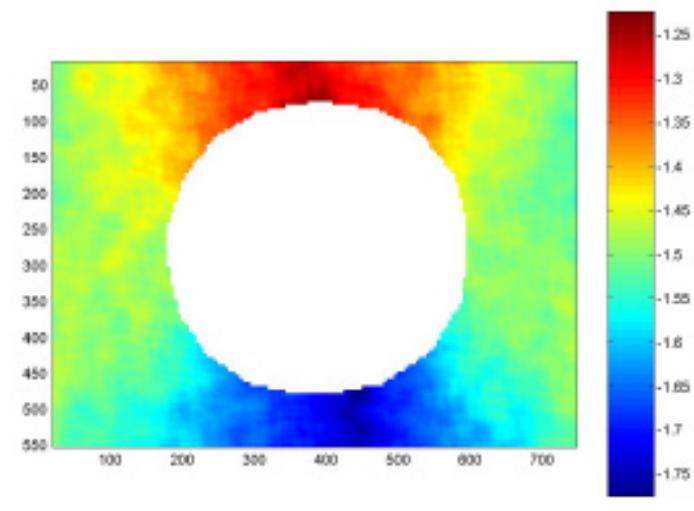

(a) Vertical displacement field at 226.1s

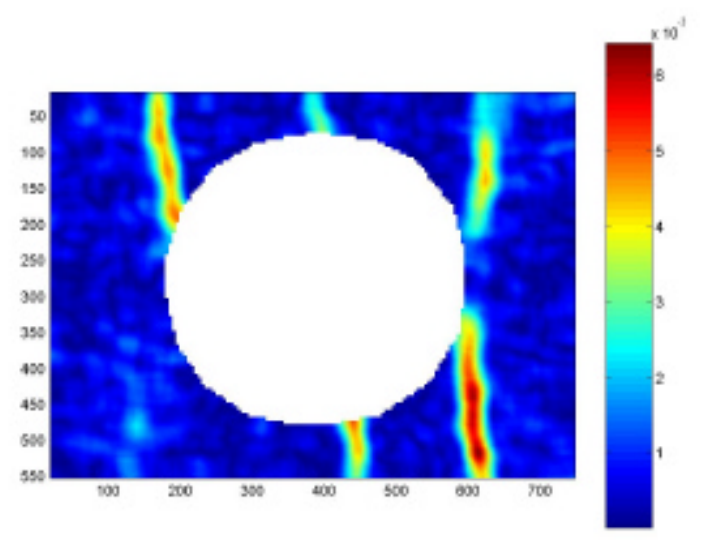

(b) Maximum shear strain field at $252.3 \mathrm{~s}$

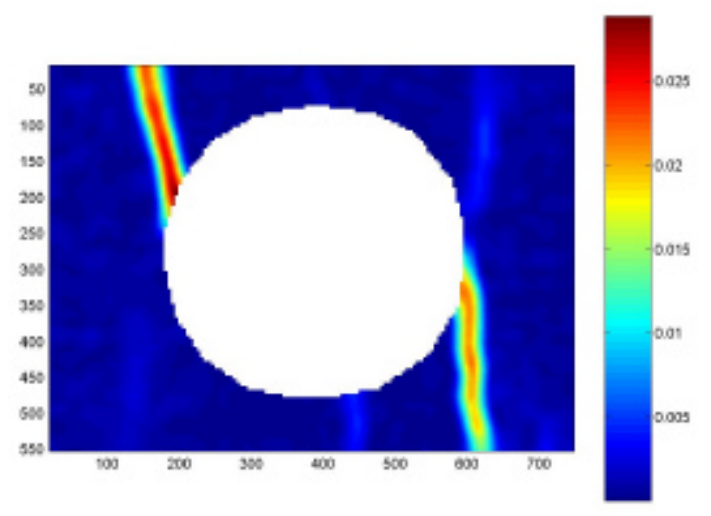

(c) Maximum shear strain field at $258.2 \mathrm{~s}$

Fig.7 Deformation field during loading (Unit for the displacement, $X$ and $Y$ axis is pixel, i.e. $0.0568 \mathrm{~mm}$ )

\section{CONCLUSION AND DISCUSSION}

The Geo-DSCM system is designed and realized. A weighted correlation equation was used to improve the accuracy on the measurement of heterogeneous deformation field, which is common to rock materials, especially on the event of failure. A data acquisition system, that is capable of capturing enough speckle images during specimen deformation and failure was constructed. The system was then used in a failure inspection experiment of a borehole rock specimen and the complicated failure procedure was revealed.

In this paper, the correlation equation was weighted according to a distance-related function. As an extension to this work, other weighting methods can be used to modify the correlation equation in the future work. If the spatial resolution of DSCM is analyzed profoundly and all these weighing methods are tested and compared, a better correlation equation may be found. However, using a weighted correlation equation is necessary for enhancing the spatial resolution of DSCM and can improve the accuracy of displacement measurement in the heterogeneous field.

In this paper, the image capturing speed is adjusted by a previous determination of the load stages. Although the stages could be determined through some preliminary test, it is still not accurate. The more appropriate way is to determine the deformation stage dynamically during loading. To realize this, it is necessary to design a real-time DSCM processing system, which analyses the deformation of rock materials and offers rough deformation information (but enough to indicate the deformation stage) during loading, using a much simpler but very fast processing algorithm. Actually, this is possible when some special techniques and a relative ly fast computer are used. With this system, the image capturing speed can be adjusted dynamically during loading, and even a second image capturing system can be trigged to concentrate on a small but more important area to obtain measurement with much higher sensitivity.

\section{ACKNOWLEDGEMENT:}

The authors thank the financial support of NSFC 40234042, College Research Foundation of Liaoning Province 202183390, and the Opening Foundation of the Key Lab of IRSM Z110304. We also appreciate the help from Prof. Jin Guanchang of Tsinghua University. 


\section{REFERENCES}

Berthaud Y, Torrenti J M, Fond C. 1997. Analysis of localization in brittle materials through optical techniques, Experimental Mechanics. 37(2): 216 220

Huang J, Chen G, Zhao Y, et al. 1990. An Experimental Study of the Strain Fields Development Prior to Failure of a Marble Plate under Compression. Tectonophysics, 175:269 284

Peters W H, Ranson W. F. 1981. Digital Imaging Techniques in Experimental Stress Analysis, Opt. Eng. 21: 427 431

Sutton M A, McNeill S R, Jang J, et al. 1988. Effects of subpixel image restoration on digital correlation error estimation. Opt. Eng. 27(10),870 877

Tian Qi, Huhns M N. 1986. Algorithms for subpixel registration. Computer vision, Graphics, and Image processing. 35:220 233

Zhao Yonghong, Liang Haihua, Xiong Chunyang et al. 2002. Deformation measurement of rock damage by digital image correlation method. Chinese Journal of Rock Mechanics and Engineering. 21(6):73 76 (in Chinese)

Zhou Peng, Goodson 2001. K E. Subpixel displacement and deformation gradient measurement using digital image/speckle correlation (DISC). Opt. Eng. 40(8):1613 1620 